\title{
Millimeter dust emission from an SMC cold molecular cloud
}

\author{
M. Rubio ${ }^{1}$, F. Boulanger ${ }^{2}$, F. Rantakyro ${ }^{3,1}$, and A. Contursi ${ }^{4}$ \\ ${ }^{1}$ Departamento de Astronomia, Universidad de Chile, Casilla 36-D, Santiago \\ e-mail:mrubio@das.uchile.cl \\ 2 Institut d'Astrophysique Spatiale, Université Paris-Sud, Bât. 121, 91405 Orsay Cedex, France \\ 3 European Southern Observatory, Casilla 19001, Santiago 19, Chile \\ ${ }^{4}$ Max-Planck-Institute fur Extraterrestische Physik (MPE), Posfach 1312, 85741 Garching, Germany
}

Received 13 April 2004 / Accepted 25 July 2004

\begin{abstract}
We presented SIMBA 1.2 mm continuum observations of SMCB1-1 an SMC molecular cloud known to be distant from massive ionizing stars. The gas mass derived from the SIMBA flux is a factor of $\sim 10$ higher than the virial mass previously reported from $\mathrm{CO}$ observations. We propose an interpretation where the virial mass underestimates the total mass because the $\mathrm{CO}$ emission comes from dense clumps which do not trace the full cloud area and velocity distribution. This work sets an important question for the understanding of star formation in low metallicity gas. Do CO observations grossly underestimate the amount of dense self gravitating clouds where stars can form?
\end{abstract}

Key words. ISM: molecular clouds - ISM: dust - ISM: individual object: LMC-SMC - infrared: ISM: continuum

\section{Introduction}

In low metallicity environments, due to the reduced shielding, $\mathrm{CO}$ emission is expected to only trace the densest parts of molecular clouds but not their diffuse envelopes which make most of the mass of Galactic Giant Molecular Clouds (Lequeux et al. 1994). In the nearest low metallicity galaxy, the Small Magellanic Cloud (SMC), this expectation is empirically supported by the reduced ratio between $\mathrm{CO}$ luminosity and virial mass compared to the Milky Way value (Rubio et al. 1993). A large fraction of the mass of low metallicity star forming clouds, might thus consist of molecular gas with no $\mathrm{CO}$ emission and atomic gas too cold to be seen in emission. Observations of dust emission in such galaxies can be a unique mean in tracing this so-far hidden matter.

We have observed several molecular clouds in the Magellanic Cloud with the $1.2 \mathrm{~mm}$ SIMBA bolometer at SEST. In this Letter we report the detection of continuum $1.2 \mathrm{~mm}$ emission towards a cold quiescent molecular cloud in the SMC, known to be distant from massive star forming regions. In this cloud no free-free emission is expected and the $1.2 \mathrm{~mm}$ emission can be assigned entirely to dust.

\section{SIMBA observations}

The $\lambda 1.2 \mathrm{~mm}$ observations were made with SIMBA, a 37 element bolometer array attached to the SEST telescope, La Silla, Chile. The HPBW of a single element is about $24^{\prime \prime}$, the separation between elements on the sky are 44".

Three observing periods were allocated for this study, 68-019, 69.C-0576, and 70.C-371. SMCB1-1 in the SMC was observed at three occasions, 29th to 30th of October 2001 the 30th of July 2002, and November 2002. The observations where done with the on-the-fly mapping mode where the instrument scans rapidly over the source $(80 \mathrm{arcsec} / \mathrm{s})$. Each map covered a 900 by 792 arcsec area in Azimuth and Elevation and the final map is a rms weighted average of 51 individual maps. These maps were reduced for each observing period and latter combined. The rms in the final image but excluding the source is $2 \mathrm{mJy} / \mathrm{beam}$.

The data reduction was done within the MOPSI package. We have followed the general procedure described in the SIMBA Handbook (http://puppis.ls.eso.org/staff/ SIMBA/manual/SIMBA/index.html). The first step was to produce the rms weighted maps without any assumption of the source structure, the second step used a polygon including the source (seen in the first step) so that the baseline fitting and the sky noise reduction excluded the source structure, and the final step was done by using the source structure obtained from the first two steps as input on the source model. The final step was repeated until no significant chnages was found between consecutive iterations.

The flux calibration was done by observing Uranus each of the observing nights to obtain the absolute flux calibration, and we calibrated each of the maps individually. We estimate that the final calibrated image has an accurancy of $\sim 15 \%$ for the absolute flux.

\section{Results}

Continuum $1.2 \mathrm{~mm}$ emission observed with SIMBA was found towards SMCB1-1 cold molecular cloud. Figure 1, left panel, shows the $1.2 \mathrm{~mm}$ SIMBA image of SMCB1-1. In the right 

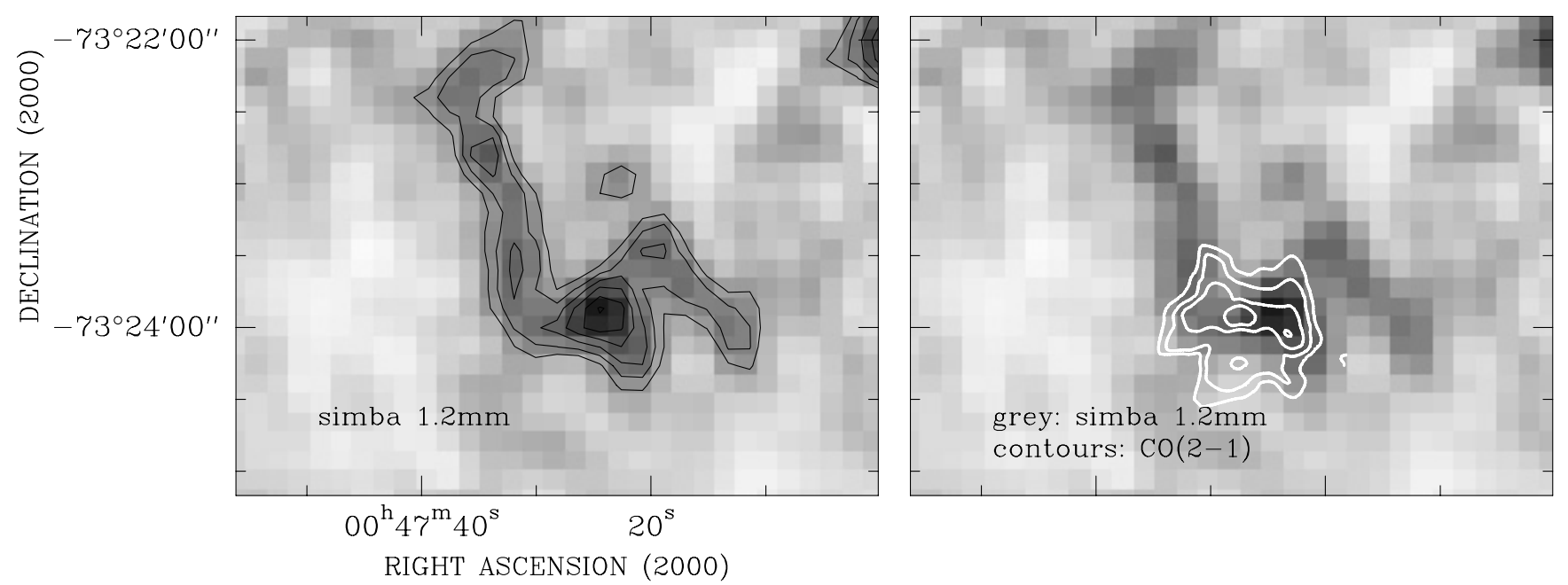

RIGHT ASCENSION (2000)

Fig. 1. Left: SIMBA image of SMCB1-1. Contour levels from 15 to $45 \mathrm{mJy} /$ beam in steps of $5 \mathrm{mJy} /$ beam. Right: CO(2-1) contours overlayed on SIMBA grey scale image (c.1.: 2.2, 3.2, 4.5, $5.4 \mathrm{~K} \mathrm{~km} \mathrm{~s}^{-1}$ ).

panel, we have superimposed the $\mathrm{CO}(2-1)$ emission line contours observed toward this source (Rubio et al. 1996). Clearly, the $\mathrm{CO}$ emission is different from the SIMBA emission. The $1.2 \mathrm{~mm}$ SIMBA flux of the $\mathrm{CO}$ cloud measured inside the $25 \mathrm{mJy}$ contour level is $50 \pm 5 \mathrm{mJy}$ while the flux interior to the first contour shown is $249 \pm 5 \mathrm{mJy}$. We determined the sky noise by taking reference areas of comparable size to estimate the mean and standard deviation outside the source. We subtracted the mean value to the integrated SIMBA flux measurements.

The continuum mm emission can arise from different mechanisms: thermal free-free from ionized gas, $1.3 \mathrm{~mm}$ $\mathrm{CO}(2-1)$ line emission, and cold dust emission. The Parkes survey at 4.8 and $8.5 \mathrm{GHz}$ (Filipovic et al. 1997) allow to put an upper limit to the free-free contribution. The radio free-free flux from $\mathrm{H}$ II regions scales with $v^{-0.1}$. With this frequency dependence, the $50 \mathrm{mJy}$ SIMBA flux translates into fluxes of 74 and $70 \mathrm{mJy}$ at 4.8 and $8.5 \mathrm{GHz}$ respectively, detectable values for the sensitivity limits of 5 and $10 \mathrm{mJy}$ (rms noise) of the Parkes survey. No radio source is detected at the SIMBA position and thus the free-free emission could account for at most $20 \%$ ( $3 \sigma$ upper lilmit) of the SIMBA flux. Additionally, no $\mathrm{H}_{\alpha}$ (Davies et al. 1976) nor $\mathrm{Br}_{\gamma}$ (Rubio, private communication) observations show emission at the position of the SIMBA source. We checked with existing SEST observations (Rubio et al. 1996) that the contribution due to the $1.2 \mathrm{~mm}$ $\mathrm{CO}(2-1)$ emission line is negligible.

SMCB1-1 has been observed with ISO. Reach et al. (2000) analyzed mid-IR spectroscopic observations showing the PAH mid-IR features and ISOCAM broad-band filter observations at $7 \mu \mathrm{m}$ and $15 \mu \mathrm{m}$. The ISO observations show diffuse emission at $15 \mu \mathrm{m}$ with a similar extent as that of the $1.2 \mathrm{~mm}$ emission and a point like source on the side of the $\mathrm{CO}$ emission which does not appear in the SIMBA map. Comparing the IR brightness with that of Solar Neighborhood clouds Reach et al. (2000) conclude that SMCB1-1 could be similar to the Ophiucus molecular cloud. The mid-IR emission could come from warm gas at the surface layers of a colder molecular cloud. But the column density at which the UV penetration would warm the gas corresponds to $N(\mathrm{H}) \sim 10^{22}(\tau=1$ in the $\mathrm{SMC}$ ), and is an order of magnitud larger than that needed to account for the $1.2 \mathrm{~mm}$ flux which comes from galactic dust in shielded regions. The $\mathrm{CO}$ emission was modelled by Lequeux et al. (1994) and they found from the CO excitation a gas temperatures of $\sim 10-15 \mathrm{~K}$. This cold molecular cloud is therefore the one detected at SIMBA wavelenghts.

Thus, SMCB1-1 shows up as a cold molecular cloud and we believe that the SIMBA observations measure emission from cold dust.

\section{Dust emission}

The IR brightness of a cloud is

$I_{v}=\kappa_{v} \sigma_{\text {dust }} B_{v}=\kappa_{v} x_{\mathrm{d}} \mu m_{\mathrm{H}} N(\mathrm{H}) B_{v}\left(T_{\mathrm{d}}\right)$

where $\kappa_{v}$ is the absorption coefficient per unit dust mass, $\sigma_{\text {dust }}$ the cloud dust mass per unit surface, $x_{\mathrm{d}}$ the dust to gas mass ratio, $\mu \mathrm{m}$ is the gas weight per $\mathrm{H}, N(\mathrm{H})$ the hydrogen column density, and $T_{\mathrm{d}}$ the dust temperature.

From Eq. (1) one gets the following relation between the cloud integrated flux $\mathrm{S}_{v}$ and the cloud gas and dust masses:

$M_{\mathrm{gas}}=\frac{S_{v} D^{2} \mu m_{\mathrm{H}}}{\epsilon_{\mathrm{d}} B_{v}\left(T_{\mathrm{d}}\right)}$

where $\epsilon_{\mathrm{d}}$ is the dust emissivity per $\mathrm{H}$, and $D$ the distance to the cloud.

The dust emissivity per $\mathrm{H}$ atom in the atomic ISM in the Solar neighborhood, determined from COBE and H I observations (Boulanger et al. 1996) is $5.0 \pm 0.9 \times 10^{-27} \mathrm{~cm}^{2}$ at $1.2 \mathrm{~mm}$ as scaled from the measured value at $1.1 \mathrm{~mm}$. This is close to the value of $3.9 \times 10^{-27} \mathrm{~cm}^{2}$ in the Draine \& Lee (1984) graphite + silicates dust model. In the Solar Neighborhood the dust to hydrogen mass ratio $\left(x_{\mathrm{d}} \times \mu\right)$ is $1 \%$ based on estimated abundances of elements in dust (Sofia \& Meyer 2001). For this dust to hydrogen mass ratio, the empirical determination of $\epsilon_{\mathrm{d}}(1.2 \mathrm{~mm})$ translates into $\kappa_{v}(1.2 \mathrm{~mm})=0.30 \mathrm{~cm}^{2} \mathrm{~g}^{-1}$. 
Table 1. Cloud masses.

\begin{tabular}{ccccc}
\hline \hline Region & $S_{1.2 \mathrm{~mm}}$ & $M_{\text {gas }}$ & $M_{\mathrm{vir}}$ & $M\left(\mathrm{H}_{2}\right)$ \\
& $\mathrm{mJy}$ & $M_{\odot}$ & $M_{\odot}$ & $M_{\odot}$ \\
\hline SMCB1-1 & $50.0 \pm 5$ & $2.7 \times 10^{5}$ & $1.2 \times 10^{4}$ & $2.7 \times 10^{4}$ \\
\hline
\end{tabular}

To calculate the gas mass we also need to estimate the dust to gas mass ratio. The SMC metallicity derived from HII region observations is $\sim 0.1$ solar (Dufour 1984). Assuming that a fixed fraction of the heavy elements is in dust we use $x_{\mathrm{d}}(\mathrm{SMC})=0.1 \times x_{\mathrm{d}}(\odot)=7 \times 10^{-4}$. The relation between the dust to gas mass ratio and the metallicty has been discussed on large scale in galaxies. Studies of spirals (James et al. 2002) and the modeling of dust formation history across the Milky Way (Dwek 1998) support a linear relation but other studies suggest that the dust abundance is more reduced than the metallicity in some blue compact and dwarf irregular galaxies (Lisenfeld \& Ferrara 1998; Galliano et al. 2003; Bot et al. 2003). This last result might apply to the diffuse medium but not necessarily to molecular clouds where one expects all heavy elements to be accreted on dust independently of the galaxy type.

To apply formula (2) and (3), we also have to assume a dust temperature. Based on a comparison of $100 \mu \mathrm{m}$ IRAS and $170 \mu \mathrm{m}$ ISOPHOT maps of the SMC, Bot et al. (2003) found a dust temperature $T_{\mathrm{d}}=22 \mathrm{~K}$ outside massive star forming regions. Within molecular clouds dust extinction attenuates the stellar radiation and the dust is expected to be colder. Temperatures of $12 \mathrm{~K}$ have been reported for molecular clouds in the Solar Neigborhood and proto-stellar condensations (Bernard et al. 1999; Stepnik et al. 2003; Bianchi et al. 2003). The temperature in SMC molecular cloud might be higher because there is less dust to attenuate it. We thus assume a slightly higher dust temperature of $15 \mathrm{~K}$. For an SMC distance, $D$, of $63 \mathrm{kpc}$ Eq. (2) becomes:

$$
\begin{aligned}
M_{\mathrm{gas}}= & 5.4 \times 10^{3} \frac{S_{v}(1.2 \mathrm{~mm})}{\mathrm{mJy}} \frac{\epsilon_{\mathrm{d}}}{4.3 \times 10^{-27} \mathrm{~cm}^{2}} \\
& \times \frac{0.1 x_{\mathrm{d}}(\odot)}{x_{\mathrm{d}}(\mathrm{SMC})} \frac{B_{v}(15 \mathrm{~K})}{B_{v}\left(T_{\mathrm{d}}\right)} M_{\odot}
\end{aligned}
$$

and for the gas column density:

$$
\begin{aligned}
N(\mathrm{H})= & 1.0 \times 10^{22} \frac{I_{v}(1.2 \mathrm{~mm})}{1.06 \mathrm{mJy} / \text { beam }} \frac{\epsilon_{\mathrm{d}}}{4.3 \times 10^{-27} \mathrm{~cm}^{2}} \\
& \times \frac{0.1 x_{\mathrm{d}}(\odot)}{x_{\mathrm{d}}(\mathrm{SMC})} \frac{B_{v}(15 \mathrm{~K})}{B_{v}\left(T_{\mathrm{d}}\right)} \mathrm{H} \mathrm{cm}^{-2} .
\end{aligned}
$$

\section{Gas mass}

Derivation of the gas mass from CO emission in low metallicity systems is dependent on the determination of the appropriate $X=M\left(\mathrm{H}_{2}\right)_{\mathrm{co}} / L_{\mathrm{CO}}$ conversion factor. The assumption of virial equilibirum between gravitational and turbulent energy have so far been the only mean to determine the $X$ factor in the SMC. Under this assumption, based on early $\mathrm{CO}(1-0)$ observations with the CTIO/Columbia $\mathrm{mm}$ telescope and the SEST, Rubio et al. (1993) found $X_{\mathrm{SMC}}$ to be size dependent and equal to $3.9 \times(R / 10 \mathrm{pc})^{-0.7} X_{\mathrm{Gal}}$ where $R$ is the cloud radius, defined as $\sqrt{(} A / \pi)$. The SMC NANTEN survey (Mizuno et al. 2001), with a linear resolution of $40 \mathrm{pc}$, found consistently an average conversion factor $X_{\mathrm{SMC}} \sim 9 X_{\mathrm{Gal}}$. The $\mathrm{CO}(1-0)$ luminosity of SMCB1-1 is $1.2 \times 10^{3} \mathrm{~K} \mathrm{~km} \mathrm{~s}^{-1}$ (Rubio et al. 1996) which multiplied by the appropriate $X$ factor gives $M\left(\mathrm{H}_{2}\right)_{\text {co }}=2.7 \times 10^{4} M_{\odot}$ in Table 1 . The cloud radius determined from the higher angular resolution 2-1 observations which better resolve it is $12 \mathrm{pc}$. We also list in the table the virial mass computed from the $\mathrm{CO}(2-1)$ velocity dispersion and the cloud radius.

The unexpected result from the SIMBA observations is that they lead to a gas mass a factor of 10 to 20 times larger than the mass derived from $\mathrm{CO}$ observations. Before discussing a possible interpretation of this difference, we review uncertainties on the different factors involved in the mass calculation.

The dust emissitivity could be higher - in molecular clouds, grains are thought to grow through coagulation into fractal aggregates with a higher far-IR/mm emissivity per mass (Ossenkopf \& Henning 1994). Several observations do suggest an enhancement by a factor $\sim 3$ in Solar Neighborhood molecular clouds with respect to the diffuse ISM value (Stepnik et al. 2001; Cambresy et al. 2001; del Burgo et al. 2003). There is some evidence that this result also applies on large scale in the Galaxy (Boulanger et al. 2002) and NGC 891 (Alton et al. 2000). This enhancement is disputed for NGC 891 by other observations and analysis (Guelin et al. 1993) and results on nearby spiral galaxies (James et al. 2003).

The main uncertainty on the gas mass appear to come from the dust emissivity. Assuming for SMCB1-1, a molecular clouds Solar Neighborhood dust emissivity rather than the diffuse ISM value, the difference between gas and virial mass is reduced from 20 to 7 but remains high. We do not see any additional reason to decrease further the gas mass estimated from the dust emission.

The remaining factors entering the mass calculation are less uncertain. We assumed a dust to gas ratio ten times smaller than in the Solar Neighborhood for the SMC based on metallicity measurements and the assumption that the same fraction of the heavy elements are in dust. To reduce the gas mass one will need a larger dust to gas ratio. In the SMC, metallicity could be higher than $1 / 10$ as derived from HII regions. Hill 1997 and Hill et al. (1997) find an iron defficiency in a sample of supergiants stars ranging from -0.59 to -0.89 dex and a mean $[\mathrm{C} / \mathrm{Fe}]=-0.3,[\mathrm{O} / \mathrm{Fe}]=-0.18$. The metals as indicated by iron seem to be slightly more abundant than $1 / 10$ Solar but not very much. An alternative possibility is that a higher fraction of Carbon and Oxygen could be on dust in the SMC than in the Solar Neighborhood. We do not see any physical reason to believe this, and if was the case it would only marginally increase the dust to gas mass ratio. Would all Carbon and Oxygen be on dust, the dust to mass ratio would be increased by a factor of less than 2.

Dust temperature - The dust could be warmer than the assumed temperature of $15 \mathrm{~K}$. The dust temperature is observed to be $22 \mathrm{~K}$ in the diffuse ISM (Bot et al. 2003). The dependence of the $\mathrm{mm}$ emission for temperatures higher than $15 \mathrm{~K}$ is only slighlty larger than linear with dust temperature. Within 
the plausible range 15 to $22 \mathrm{~K}$, a higher dust temperature will thus barely reduce the gas mass. We exclude a significant contribution to the SIMBA flux from a layer of warmer dust heated by non-ionizing B stars associated with the cloud. The column density of such a heated cloud layer would correspond to the $\mathrm{UV}$ penetration depth and thus $\tau_{\mathrm{UV}} \sim 1$ which translates into $N(\mathrm{H}) \sim 10^{22} \mathrm{H} \mathrm{cm}^{-2}$ for the SMC dust abundance. Using formula (4) we computed that for a dust temperature of $50 \mathrm{~K}$ such a layer would account only for $10 \%$ of the peak $1.2 \mathrm{~mm}$ brightness of $45 \mathrm{mJy} / \mathrm{beam}$. Higher dust temperatures on the scales resolved by these observations (the $24^{\prime \prime}$ beam corresponds to $7 \mathrm{pc}$ ) are precluded by the absence of very luminous stars. Dust is expected to be warmer than $50 \mathrm{~K}$ only within $\sim 0.1 \mathrm{pc}$ of a few $10^{3} L_{\odot}$ star.

In the SMC due to the low metallicity and dust content, the $\mathrm{CO}$ emission is thought to arise only from dense high column density clumps (Lequeux et al. 1994). For a density of $10^{4} \mathrm{H} \mathrm{cm}^{-3} \mathrm{CO}$ becomes dominant for column densities $>2 \times 10^{22} \mathrm{H} \mathrm{cm}^{-2}$. Thus, $\mathrm{CO}$ does may not trace all of the cloud volume and gas velocity distribution and thereby the virial mass deduced from the $\mathrm{CO}$ line width and emitting area is only a lower limit for the mass of SMC molecular clouds.

Thus, our interpretation of the mass difference is that the SIMBA observations are detecting emission from an extended envelope of $\mathrm{HI} / \mathrm{H}_{2}$ gas with a density lower than that of the $\mathrm{CO}$ emitting gas.

\section{Conclusion}

We presented SIMBA $1.2 \mathrm{~mm}$ continuum observations of SMCB1-1 an SMC molecular cloud known to be away from massive ionizing stars. The conclusions from this Letter are:

- The $1.2 \mathrm{~mm}$ flux is measuring emission from cold dust.

- Within the uncertainities in the dust emissitivity, the gas mass derived from the SIMBA flux is a factor of 7 to 20 times higher than the mass reported from CO observations.

- We propose an interpretation of this difference where the gas mass derived assuming virialization underestimates the total mass because the $\mathrm{CO}$ emission comes from dense clumps which do not trace the full cloud area and velocity distribution.

This work sets an important question for the understanding of star formation in low metallicity gas. Do CO observations grossly underestimate the amount of dense self gravitating clouds where stars can form? Observations of a larger number of clouds is required to answer this question on a statistical basis.

Acknowledgements. We acknowledge C. Bot for helping us with the figure and manuscript preparation. F.B. acknowledges the kind invitation received from the Chilean Center for Astrophysics. M.R. is supported by the Chilean Center for Astrophysics FONDAP No. 15010003.

\section{References}

Alton, P., Xilouris, E., Bianchi, S., et al. 2000, A\&A, 356, 795

Bernard, J. P., Dobashi, K., \& Momose, M. 1999, A\&A, 350, 197B

Bianchi, S., Goncalves, J., Albrecht, A., et al. 2003, A\&A, 399, L43

Bot, C., Boulanger, F., Lagache, G., Cambrésy, L., \& Egret D. 2004, A\&A, 423, 567

Boulanger, F., Bourdin, H., Bernard, J.-P., et al. 2002, EAS Pub. Ser., 4,151

Boulanger, F., Abergel, A., \& Bernard, J.-P. 1996, A\&A, 312, 256

Cambresy, L., Boulanger F., Lagache, G., et al. 2001, A\&A, 375, 999

Davies, R., Elliot, K., \& Meaburn, J. 1976, MemRAS, 81, 89

del Burgo, C., Laureijs, R. J., Abrahám, P., et al. 2003, MNRAS, 346, 403

Draine, B. T., \& Lee, H. M. 1984, ApJ, 285, 89

Dufour, R. J. 1984, in Structure and Evolution of the Magellanic Clouds (Dordrecht: D. Reidel Publishing Co.), IAU Symp., 108, 353

Dwek, E., Arendt, R. G., Fixsen, D. J., et al. 1997, ApJ, 475, 565

Filipovic, M., Jones, P., White, G., et al. 1997, A\&AS, 121, 321

Galliano, F., Madden, S., Jones, A., et al. 2003, A\&A, 407, 159

Guelin, M., Zylka, R., Mezger, P. G., et al. 1993, A\&A, 279, L37

Hill, V. 1997, A\&A, 324, 435

Hill, V., Barbuy, B., \& Spite, M. 1997, A\&A, 323, 461

James, A., Dunne, L., Eales, S., et al. 2002, MNRAS, 335, 753

Lequeux, J., Pineau de Forest, G., Roueff, E., et al. 1994, A\&A, 292, 371

Lisenfeld, U., \& Ferrara, A. 1998, ApJ, 496, L145

Mizuno, N., Rubio, M., Mizuno, A., et al. 2001, PASJ, 53, 45

Ossenkopf, V., \& Henning, Th. 1994, A\&A, 291, 943

Rantakyro, F., Rubio, M., Johansson, L. E. B., et al. 2004, A\&A, in preparation

Reach, W., Boulanger, F., Contursi, A., et al. 2000, A\&A, 361, 895

Rubio, M., Lequeux, J., \& Boulanger, F. 1993, A\&A, 271, 9

Rubio, M., Lequeux, J., Boulanger, F., et al. 1996, A\&AS, 118, 263

Sofia, U. J., \& Meyer, D. M. 2001, ApJ, 554, L221

Stepnik, B., Abergel, A., \& Bernard, J.-P. 2003, A\&A, 398, 551 